\title{
Isolated Cecal Rupture after Blunt Abdominal Trauma
}

\author{
Alemayehu Ginbo Bedada, ${ }^{1}$ Elijah Wade Riddle, ${ }^{2}$ Alemayehu Bekele Eshetu, ${ }^{3}$ Georges Azzie ${ }^{4}$ \\ 1. Department of Surgery, University of Botswana, Faculty of Medicine, Gaborone, Botswana \\ 2. Department of Surgery, University of Pennsylvania, Philadelphia, USA \\ 3. Department of Pathology, University of Botswana, Faculty of Medicine, Gaborone, Botswana \\ 4. Division of General and Thoracic Surgery, Hospital for Sick Children, Toronto Canada
}

\section{Correspondence to: Dr. Alemayehu Bedada; email: bedale00@yahoo.co.uk}

\section{Summary}

Cecal perforation following blunt abdominal trauma is an uncommon and challenging injury. We report a 19 -yearold HIV-positive woman who presented with abdominal pain after a high-speed motor vehicle crash. Abdominal exam revealed a seatbelt sign with evidence of peritonitis; Focused Assessment with Sonography for Trauma showed free intraperitoneal fluid. After fluid resuscitation and antibiotics, the patient was taken for urgent laparotomy. Intraoperatively, we discovered hemoperitoneum and an isolated rupture of the cecum. A right hemicolectomy with end-to-end ileo-transverse colon anastomosis was performed. Her only significant postoperative complication was a superficial wound infection. We review the epidemiology of hollow viscus injury in blunt trauma and discuss important

\section{Introduction}

Hollow viscus injury (HVI) following blunt trauma is challenging and uncommon, presenting in $0.3-9 \%$ of cases (1-7). HVI may involve serosal lacerations, mural hematomas, or ruptures following an acute rise in intraluminal pressure $(1-4,8)$. Reported rates of HVI following blunt trauma vary by organ: small bowel is affected in $55.0-80.9 \%(1-3,5)$, colon in $10.0-17.0 \% \quad(1,5,8)$, duodenum in $10-12 \%(1,5)$, rectum in $7.0 \%(1)$, stomach in $3.0-4.3 \%(1,5)$, cecum in $0.57 \%$ (2), and appendix in $0.4 \%$ (5) of cases. HVI with perforation occurs in less than $1.0 \%$ of patients with blunt abdominal trauma. $(4,5,7)$

Although compression of the intestine between the abdominal wall and the spine is considered the primary mechanism of injury $(1,2,6-8)$, a combination of factors is probably involved. About $25 \%$ of patients who have surgery for presumed HVI have more than one site of bowel injury (2) and $21.4 \%$ have associated solid visceral injury (2). Seatbelts reduce mortality but are thought to increase HVI, particularly when worn incorrectly (6).

Abdominal pain and tenderness are common findings, but physical examination alone is not accurate in dictating considerations in diagnosis and treatment.

Keywords: Blunt abdominal trauma, Cecal rupture, HIV positive, Hollow viscus

Ann Afr Surg. 2020; 17(3):134-136

DOI: http://dx.doi.org/10.4314/aas.v17i3.11

Conflicts of Interest: None

Funding: None

(C) 2020 Author. This work is licensed under the Creative Commons Attribution 4.0 International License

Submitted: 4 August 2019

Revised: 9 December 2019

Accepted: 28 January 2020

Online first: 29 May 2020

the need for surgery (1,9). Associated injuries, administered pain medications, and alcohol or drug intoxication reduce the reliability of clinical examination $(1,9)$. Bruising of the abdomen in the pattern of the seatbelt, the so-called "seatbelt sign," is indicative of significant transfer of energy and highly associated with intra-abdominal injury (7), more so when free intraabdominal fluid or fracture of thoracolumbar vertebrae is present (6).

No individual or combination of diagnostic tests, including physical examination, x-ray, ultrasound, and computed tomography $(1,4,8)$, have been shown to accurately detect colonic injury (4). Certain CT findings including focal wall thickening, bowel wall hematoma, enteral contrast extravasation, discontinuity of bowel wall, foci of air near a hollow viscus, frank pneumoperitoneum, and intraperitoneal fluid suggest HVI $(1,2,4,6,7)$.

Simple closure may be appropriate in the case of an isolated perforation with minimal contamination $(2,10)$. Resection and anastomosis are required for some small and large bowel injuries $(2,10)$. A proximal diverting 
stoma may be of value in intestinal injuries where the patient is toxic or moribund, or when a grossly contaminated peritoneum is present $(2,10)$.

\section{Case report}

A 19-year-old girl presented with abdominal pain following a motor vehicle crash. She was a restrained backseat passenger in a car that struck a tree at high speed. The driver died and two other passengers sustained major head and abdominal injuries. On primary survey, her airway was intact and she communicated in full sentences. A cervical collar was placed. She was breathing comfortably at 16 breaths/min with bilateral breath sounds. Oxygen saturation was $100 \%$ on room air. She had a pulse rate of 101/min with blood pressure of 148/112 $\mathrm{mmHg}$. Femoral and peripheral pulses were palpable with normal capillary refill. Two peripheral intravenous cannulas were placed, and fluids initiated after drawing blood for labs. Glasgow Coma Scale was 15 with no neurologic deficits. FAST showed free intraperitoneal fluid. Chest and pelvic radiographs were normal.

Secondary survey showed mild abdominal distension, seatbelt sign across the right lower abdomen, and tenderness to percussion with guarding. Lab results showed hemoglobin of $12.9 \mathrm{mg} / \mathrm{dL}, \mathrm{WBC}$ of $3,370 / \mu \mathrm{L}$, and platelet count of $101,000 / \mu \mathrm{L}$.

The patient had been diagnosed with HIV 18 months before the accident and started on antiretroviral treatment, but had defaulted in the previous two months. At the time of presentation, her CD4 count was low at $465 / \mu \mathrm{L}, \mathrm{CD} 8$ was $685 / \mu \mathrm{L}$, and viral load was high at $1,218 \mathrm{cop} / \mathrm{mL}$.

She received IV antibiotics and crystalloid fluids. She was taken for exploratory laparotomy approximately 8 hours after the accident, as another passenger required surgery more urgently. Hemoperitoneum and fecal contamination were encountered on opening the abdomen. The cecum was ruptured anteriorly involving part of its base. It was about $5 \mathrm{~cm}$ in diameter, non-bleeding, edematous, ragged and dusky edge, and very close to ileocecal valve. All other organs were uninjured. The surgeon performed a right hemi-colectomy with primary ileo-transverse colon anastomosis (Fig. 1). The incision was partially closed with staples about $1.5 \mathrm{~cm}$ apart.

The postoperative course was notable for fevers, ultimately attributed to a superficial wound infection which was managed with local wound care after removing a few staples. She started liquids on postoperative day 3 and progressed to soft foods on day 6. She was discharged on day 19 , with ongoing wound care at a nearby clinic. She was followed for two months and had no major complaints.
Histologic examination of the specimen showed focal sloughing of the mucosa, without ulceration or significant inflammation. There was marked vascular congestion, edema and haemorrhage in the submucosa with focal areas of necrosis. Gram stain, modified Gomori methenaminesilver nitrate stain and Ziehl-Neelsen stains failed to show any infectious agent. The mucosa-associated lymphoid tissue and underlying muscle coat were intact. No suppurative or granulomatous inflammation or neoplastic cell infiltration were noted throughout the layers of the gut (Fig. 2).

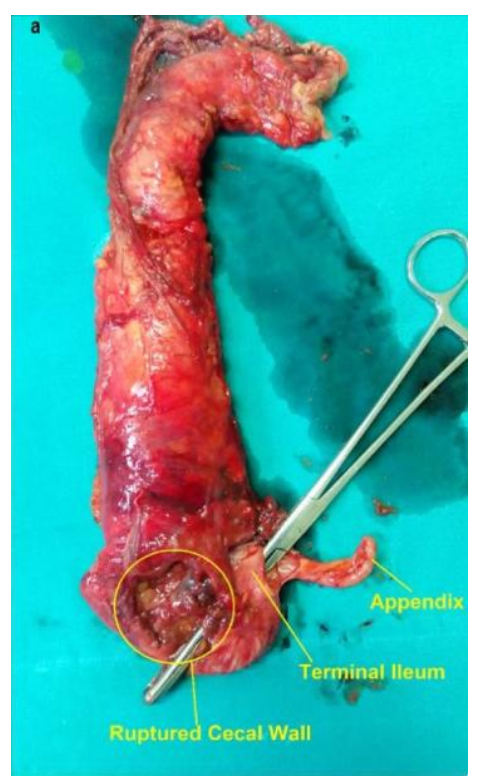

Figure 1. Right hemi-colectomy specimen with ruptured cecum.

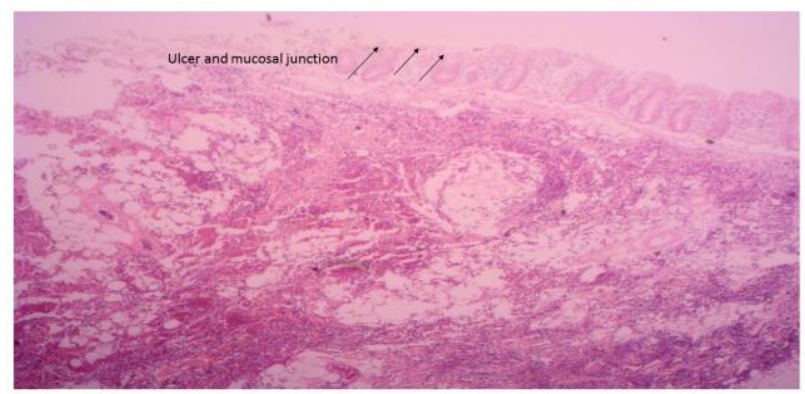

Figure 2. Histology slide revealing no underlying disease process or anatomic abnormality.

\section{Discussion}

Trauma is the leading cause of death in young adults $(2,4,9)$. The most common cause of blunt trauma is road traffic crash $(2,6,8.9)$; however, isolated hollow viscus rupture in blunt trauma is very rare. Our patient sustained a rare full-thickness cecal injury, which is infrequently reported in literature. Perforating HVI following minor blunt trauma has been attributed to underlying disease processes, spontaneous perforation in immune compromised patients, and congenital segmental bowel wall defects $(3,11,12)$. Although our patient was immune 
compromised (HIV positive, off anti-retrovirals, with low CD4 count), the possibility of spontaneous or underlying disease causing the cecal rupture seems unlikely. Rather, significant bruising on the abdomen suggested direct force from trauma as the cause. This was confirmed by pathology showing acute traumatic changes without underlying abnormality.

Various publications describe the potential role of different maneuvers to diagnose HVI following blunt trauma. Physical examination, abdominal x-ray, ultrasound, computed tomography, and diagnostic laparoscopy may each contribute to the diagnosis, with varying sensitivity and specificity $(1-5,7,12)$. Our patient had evidence of major intra-abdominal injury from history and physical examination, with findings of frank peritonitis. Further, FAST showed fluid in the peritoneal cavity. Cervical spine, chest, and pelvic x-rays were unremarkable, and no other investigations were performed.

We found only two prior case reports of cecal perforation following blunt trauma. Both patients died, one following cecal repair (cause of death not described) (2) and the other following right hemicolectomy (death reportedly due to continuing sepsis and multiple organ failure) (12). Our patient survived after right hemicolectomy, which was necessary given the large, contaminated defect near the ileocecal valve.

Overall, reported mortality for HVI was $12.7-19.8 \%(2,5)$. Mortality following colon injury was reported as 18.9$19.4 \%(4,5)$. Mortality increases with increased number of associated injuries $(2,4,5)$. In patients with HVI, the most commonly injured solid organ is the liver, while skeletal injuries dominate the associated extra-abdominal injuries $(2,8)$. Our patient had neither.

The most important factors for patient survival after HVI are early recognition and prompt surgery $(2,12)$. Delay between presentation and laparotomy has been shown to increase both morbidity and mortality $(1,2,4-9,12)$. An interval of greater than 5 hours increases the death rate 3 fold $(1,8)$. Our patient had a delay of 8 hours between accident and operation but escaped with only a superficial wound infection despite the delay and her immune compromised state.

The overall complication rate for HVI is $21.8-30.2 \%$, with highest rates for colon and rectum injuries at 32.3-36.0\% $(4,5)$. Associated complications include anastomotic leak, burst abdomen, wound infection, deep abscess, renal failure, prolonged ileus, pneumonia, and multiple organ failure $(2,4,5,8)$. In addition to prompt diagnosis and treatment, postoperative management, including broadspectrum antibiotics, nutritional support, and treatment of co-morbid disease, is similarly important in preventing and managing complications (12). Average reported hospital stay after colonic injury is 17.3 days (4).

\section{Conclusion}

Isolated hollow viscus rupture after blunt abdominal trauma is rare. An even rarer subtype is isolated cecal rupture. Early surgical treatment is critical in the management of these patients and should not be delayed for further investigations if suggestive clinical features are present $(2,11)$. Our patient's outcome suggests that HIVpositive patients with low CD4 count may be managed like any other patient. Finally, though our patient demonstrates that the forces of blunt trauma may be sufficient to cause isolated perforation in an anatomically-normal cecum, care must be taken to rule out underlying pathology that may predispose to cecal injury.

\section{References}

1. Joseph DK, Kunac A, Kinler RL, et al. Diagnosing blunt hollow viscus injury: is computed tomography the answer? Am J Surg. 2013;205(4):414-418.

2. Jha NK, Yadav SK, Sharma R, et al. Characteristics of hollow viscus injury following blunt abdominal trauma; a single centre experience from Eastern India. Bull Emerg Trauma. 2014;2(4):156-160.

3. Onwubiko C, Pennington EC, Mooney DP, et al. Intestinal perforation due to minor blunt abdominal trauma - a harbinger of underlying disease pathology. J Pediatr Surg Case Reports. 2015;3(1):35-37.

4. Williams MD, Watts D, Fakhry S. Colon injury after blunt abdominal trauma: results of the EAST Multi-Institutional Hollow Viscus Injury Study. J Trauma. 2003;55(5):906-912.

5. Watts DD, Fakhry SM. EAST Multi-Institutional Hollow Viscus Injury Research Group. Incidence of hollow viscus injury in blunt trauma: an analysis from 275,557 trauma admissions from the East multi-institutional trial. J Trauma. 2003;54(2):289-294.

6. Gönüllü D, Ilgun S, Gedik ML, et al. Gastrointestinal injuries in blunt abdominal traumas. Chirurgia (Bucur). 2015;110(4):346-350.

7. Jost E, Roberts DJ, Penney T, et al. Accuracy of clinical, laboratory, and computed tomography findings for identifying hollow viscus injury in blunt trauma patients with unexplained intraperitoneal free fluid without solid organ injury. Am J Surg. 2017;213(5):874-880.

8. Goettler CE, Rotondo M. Blunt colon trauma. Sem Colon Rectal Surg. 2004 15(2):105-111.

9. Garner J. Blunt and penetrating trauma to the abdomen, Surgery (Oxford). 2005;23(6):223-228.

10. Townsend, CM, Beauchamps RD, Eds. Sabiston textbook of surgery: the biological basis of modern surgical practice. Philadelphia, PA: Elsevier Saunders; 2012.

11. Tamai M, Satoh M, Tsujimoto A. Segmental muscular defects of the intestine: a possible cause of spontaneous perforation of the bowel in adults. Hum Pathol. 2013;44(12):2643-2650.

12. Alexander P, Schuman E, Vetto RM. Perforation of the colon in the immunocompromised patient. Am J Surg. 1986;151(5):557-561 